\title{
Photonics West 2014
}

\author{
The convergence of micro-optics and microelectromechanical systems continues to spawn new \\ directions in optics.
}

Despite the unusually dry conditions in much of California, as evidenced by the record low snow levels and talk of water shortages in various municipalities in the San Francisco Bay area, the heavens opened rather consistently for the duration of SPIE's 2014 Photonics West conference, which was held over 1-6 February 2014 at the Moscone Center in downtown San Francisco. However, the inclement weather did nothing to dampen the mood at the conference, which has now very evidently become one of the biggest events in the photonics calendar.

The fact that the meeting was big gargantuan might be a more apt description - became abundantly clear on being handed the registration package. The programme was over 430 pages long, and it did not even include abstracts. Furthermore, the package also contained an exhibition guide to help delegates navigate their way through two very packed exhibition halls. The sheer size of such a large event can be a blessing or a curse, depending on what you are hoping to get out of the meeting, but there was no shortage of choices. The BiOS symposium tackled biophotonics. LASE, as you can probably guess, hosted presentations mainly related to laser development and applications. OPTO included some work on lasers, but was more general than LASE, with greater emphasis on other optical phenomena, physics, materials and devices. There was also a host of industry-related sessions.

A field that shone particularly brightly at the meeting was the marriage of microoptics to microelectromechanical systems (MEMS), resulting in what is known as micro-opto-electromagnetical systems (MOEMS). One of the great successes in this field in recent years has been the optical applications of spatial light modulators based on micromirrors. Experts in this and related areas shared their latest insights at the MOEMS-MEMS symposium.

According to David Dickensheets, Professor of Electrical and Computer Engineering at Montana State University and symposium chair for MOEMS-MEMS at the 2014 Photonics West meeting, the sessions brought together researchers who are pushing the frontiers of nano- and microfabrication techniques and researchers whose focus is on new devices and applications.

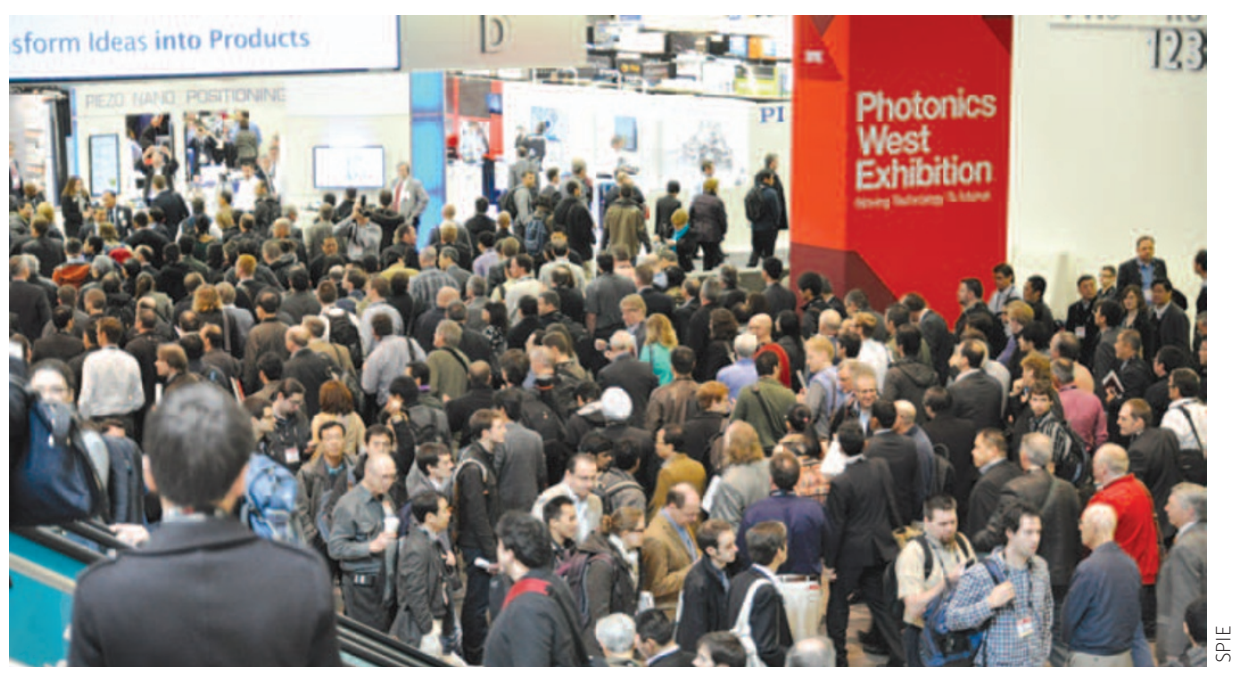

"We continue to see an emphasis on new materials, as MEMS and MOEMS expand beyond silicon fabrication technology," Dickensheets explained to Nature Photonics. "Polymers, in particular, are finding increasing use in MEMS and MOEMS, where flexibility, a large range of motion and low cost are driving considerations. Our plenary speaker John Rogers from the University of Illinois discussed the challenges of making microtechnology compatible with biology; flexible electronics that make liberal use of polymers is part of the solution to that puzzle."

According to Dickensheets, a hot topic to watch is the application of nonlinear microscopy techniques to print structures with sizes well below the diffraction limit of the writing beam. This is possible thanks to MOEMS deformable mirrors and spatial light modulators, which enable high-quality beam shapes for microscopy and lithography deep within samples.

"Plenary speaker Roger Howe of Stanford University reminded us that nanoelectromechanical systems can help solve some difficult problems, including the high leakage currents of ever shrinking CMOS transistors," Dickensheets commented. "Cornelia Denz [another plenary speaker] from Westfälische Wilhelms-Universität Münster showed how the combination of microfluidics and three-dimensional structured light beams is a powerful tool for probing and manipulating biosamples. Finally, we saw many papers in 2014 showcasing the myriad of applications for MEMS spatial light modulators, especially Texas Instruments' digital micromirror device."

What can we expect in the future? Dickensheets perceives a continuing emphasis on polymeric and other novel materials, which will enable new architectures for MEMS and MOEMS devices. In 2015, a joint session will be added between two of the conferences (that on micromachining and microfabrication process technology and that on MOEMS and miniaturized systems), which will feature new techniques and applications for polymer MEMS. This is an interesting development, because tunable micro-optic devices such as variable-focus lenses have emerged as a strong application of polymer membrane devices.

MOEMS-MEMS also attracts papers on biomedical applications of the technology a trend that is expected to continue in the future. MOEMS-MEMS and BIOS actually had two joint sessions this year: one focused on endoscopic microscopy and the other on biomedical imaging using digital micromirror devices and other MEMS micromirrors. According to Dickensheets, we can anticipate even more joint sessions between these symposia, "as MOEMS and MEMS continue to influence how we sense and interact with biological systems, including humans." 